\title{
Self-similarity principle: the reduced description of randomness
}

\author{
Raoul R. Nigmatullin, José Tenreiro Machado, Rui Menezes
}

\begin{abstract}
:
A new general fitting method based on the Self-Similar (SS) organization of random sequences is pre- sented. The proposed analytical function helps to fit the response of many complex systems when their recorded data form a self-similar curve. The verified SS principle opens new possibilities for the fitting of economical, meteorological and other complex data when the mathematical model is absent but the re- duced description in terms of some universal set of the fitting parameters is necessary. This fitting function is verified on economical (price of a commodity versus time) and weather (the Earth's mean temperature surface data versus time) and for these nontrivial cases it becomes possible to receive a very good fit of initial data set. The general conditions of application of this fitting method describing the response of many complex systems and the forecast possibilities are discussed.
\end{abstract}

PACS (2008): 92.60.hv, 89.65.Gh, 05.45.Df, 89.75.Da, 89.75.Fb

Keywords: self-similar(fractal) processes • solutions of the functional equations • complex systems • fit of economical and weather data series

\section{Introduction and formulation of the problem}

The basic purpose of any scientific research is establishing new and strongly-correlated relationships existing between two (or more) variables. In fact, all these fundamen-

tal relationships (laws) constitute the basis of any natural science. That is why any researcher in the modern world is trying to establish unknown regularities (rules) existing in nature, between strongly-correlated variables. Recently it has become more evident that with the increasing complexity of organization of the matter on different stages of its evolution, the fundamental and simple (from the mathematical point of view) rules that can exist in some complex systems are very difficult to observe and to justify. In particular, this comment can be referred totally to analysis of space-dynamic properties of different com- 

plex systems which require a holistic approach for their description. Different methods which are based on the extraction of additional information from complex systems, which have a different nature, are collected in a recent review [1], amongst other papers [2-4]. In many cases these relationships are diffusive and covered by the influence of other uncontrollable factors known in measurements as an influence of a "noise". These uncontrollable factors can completely hide a relationship which otherwise exists and therefore in many cases these factors play a destructive role. Besides these basic relationships there are other general rules that can characterize the degree of correlation between self-similar (strongly-correlated) temporal sequences. The brightest example of such kind is the relationship found by $\mathrm{H}$. E. Hurst [5]. He showed that in many processes, for some current observation period, $\tau$, the ratio between the normalized range, $R(\tau)$, and the standard deviation, $S(\tau)$, obeys to a simple power-law relationship:

$$
\frac{R(\tau)}{S(\tau)}=(\alpha \tau)^{H}, 0<H<1 .
$$

Here $\alpha$ denotes a constant and $H$ defines the Hurst powerlaw exponent. Many self-similar (fractal) series follow to this relationship, irrespective to the complexity of the natural model, where this relationship has been definitely recognized. This idea has been expanded upon in the book [6]. From another side, the idea of self-similarity is used to identify a "universal" function that describes a relatively wide class of real random sequences [7]; being the sequence of the ranged amplitudes (SRA) determined in [7] as the rank plot. It is obtained from the initial sequence when all amplitudes $\left(y_{1}>y_{2}>\cdots>y_{N}\right)$ are located in descending order for detrended random sequences having relatively large number of points (i.e. $N \geq 1000$, where $N$ defines the volume of the given sampling). For many real cases (e.g., medical, economical, weather, and other data series) that were analysed, the desired envelope of detrended sequences is described by the function containing at least two-power law exponents

$$
R(\tau)=a_{1} \tau^{v_{1}}+a_{2} \tau^{v_{2}}
$$

Here the current time, $\tau\left[\equiv \tau_{j}(j=1,2, \cdots, N)\right]$, is associated with the length of the discrete random sequence having $N$ discrete points.

The calculated fitting parameters of this function $\left(a_{1}(f), a_{2}(f), v_{1}(f), v_{2}(f)\right)$ with respect to an influence of some external factor $(f)$ (under $f$ one can imply any controllable factor, such as temperature, pressure, or otherwise) can be analysed for calculation of the desired calibration curve, or for quantitative comparison these fitting parameters with each other. Relationship (2) generalizes the Hurst relationship (1) having one power-law exponent and reflects also the self-similar behaviour of the ordered amplitudes forming the randomness. So, one can say that for many random sequences the rank plot reflects some general relationships of the second type. This idea has been used to show that for strongly-correlated systems having a memory, there is also a "universal" statistical distribution which is expressed in the form of the beta-distribution function [8]. The understanding of the meaning of many distributions helps to find additional relationships that can bond the values in some natural relationship and understand them more deeply, especially some peculiarities of complex systems where they are not known [1]. Consequently, the SS principle as a basic idea can open new possibilities in the behaviour of different complex systems and merits further research.

From another perspective, during three decades of intensive research it became clear that our world is presumably fractal in a general sense, associated with its generalizations as multifractals and random fractals of different kinds. These objects repeat themselves (randomly or regularly) on different scales, both in space and time filling an intermediate range of scales between the length of an elementary cluster and elementary physical volume associated in mathematical physics with a point. Nevertheless, nowadays it is not sufficient to say that the object/system studied has self-similar properties. It is necessary to find the fractal dimension, to determine the limits of applicability of the scaling properties and to prove the evidence/absence of log-periodic oscillations [9] that accompany any scaling process in time or space. This same phenomena was discovered in random economical and financial activities (see [10, 13-19], more references can be found in the review [1], and in papers [2-4]). Nowadays research in this field simply supposes, or postulates, the existence of scaling properties of the system studied. Also, sometimes a researcher can adduce some unjustified suppositions [6] in order to see a complex system as a fractal. Nevertheless, if the researcher did not make this initial supposition then he must find clear and justified evidences that the system really has scaling/self-similar, or fractal properties.

How to find the corvincing arguments for the sceptical scholar if a scientist has only a set of numerical data characterizing the response of a complex system and nothing else? This paper continues on the ideas discussed above, and work will be presented towards the proof that based on the justified (for many random sequences) SS principle - it is possible that many sets of random data have a self-similar structure, and thereby, the inverse problem becomes solvable for these sets. It implies to reveal 
the desired fitting function that enables to fit many selfsimilar and random functions satisfying the SS principle. The fitting parameters of this function can be used for comparing two or more random data series with each other. Besides this important peculiarity, the emerging function being continued to the future can be used for forecasting (prediction) purposes if this SS property is conserved also on some segment of time that describes a future event. We suppose that this novel description of a random curve can open new possibilities in a broader understanding of general features that can be used to characterize behaviours of different complex systems.

Having these concepts in mind the paper is organized as follows. Section 2 describes a general algorithm that can be used as the solution of the irverse SS problem. The consideration of more complex hypotheses is given in the Mathematical Appendix 5. Sections 3 and 4 are associated with real analysis of data. In order to dissipate the doubts of a potential reader we retrieved the admissible and reliable data from reliable Internet resources. Finally. Section 5 formulates the basic results of this paper and outlines the directions that can serve as an "embryo" of further research.

\section{General description of the algo- rithm}

It is known that with any one dimensional process [5] having the SS property should satisfy to the following scaling equation:

$$
S(z \xi)=\lambda_{1} S(z)+s 0,
$$

where $S(z)$ defines a physical value that depends on the argument $z$ which, in general, can accept real or complex value and can be associated with any current variable as time, frequency, coordinate or other. In general, it can accept real or complex value. The parameters $\xi$ and $\lambda_{1}$ denote the scaling factors. The constant $s_{0}$ represents a possible shift. The solution of the functional equation (3) can be written as:

$$
S(z)=A_{0}+z^{v} \operatorname{Pr}(\ln z), \quad v=\frac{\ln \left(\lambda_{1}\right)}{\ln \xi}, A_{0}=\frac{s_{0}}{1-b} .
$$

Here and below the notation $\operatorname{Pr}(\ln z)$ defines an unknown log-periodic function having a period $\ln (\xi)$, which can be presented approximately by the finite segment of the infinite series

$$
\begin{aligned}
& \operatorname{Pr}(\ln z)=A_{0}+\sum_{k=1}^{K}\left[A c_{k} \cos \left(2 \pi k \frac{\ln z}{\ln \xi}\right)+A s_{k} \sin \left(2 \pi k \frac{\ln z}{\ln \xi}\right)\right] . \\
& \operatorname{Pr}(\ln z \pm \ln \xi)=\operatorname{Pr}(\ln z) .
\end{aligned}
$$

The problem can be formulated as follows. It is necessary to justify the self-similar structure of the random data characterizing the behaviour of the complex system under analysis. Then it is necessary to develop a procedure for the fitting of the data to the function $S(z)$, to find the desired fitting parameters, $\lambda_{1}, \xi, s_{0}$, and to restore the functional equation (3) for $S(z)$ figuring in (4). Usually, the scaling equation (3) is supposed to be known a priori and, in this case, the solution (4) is restored easily. However, if we have only some data and any additional information is absent then restoration of the scaling equation (3) poses a problem. That is the reason why this problem can be formulated as inverse problem that proves and describes the self-similar properties of random signals starting from the analysis of the initial data points. To the authors best knowledge, adequate algorithms for solving imverse problems associated with description (or fitting) of self-similar random functions were not proposed in the current scientific literature and does not feature in the recent review [1]. In this paper we want to demonstrate how to develop a simple and reliable algorithm that helps to solve the inverse problem, providing a reliable fit of the random function under consideration and in the form of the scaling equation of the type (3).

\subsection{Reduction an interval to three incident points}

Let us choose some interval $\left[x_{0}, x_{k-1}\right]$ containing a set of $k$ data points $\left\{\left(x_{0}, y_{0}\right), \ldots,\left(x_{k-1}, y_{k-1}\right)\right\}$. One can reduce this information into three incident points if the first point is associated with the mean value of the amplitudes and the other two points are associated to their maximal and minimal values, correspondingly.This selection represents the simplest reduction of the given set of $k$ random points to three characteristic points $p_{1}=\operatorname{mean}\left\{y_{0}, \ldots, y_{k-1}\right\}$, $p_{2}=\max \left\{y_{0}, \ldots, y_{k-1}\right\}, p_{3}=\min \left\{y_{0}, \ldots, y_{k-1}\right\}$. It is supposed that for any finite set of $k$ data points these three incident points exist. In general, these chosen sets can contain different number of points. For the case of unequal intervals the number of selected points should be chosen based on some criterion that has a specific characteristic depending on the data under analysis. Figures 1-4 demonstrate the result of this specific reduction when adopting equal length intervals. In order to limit the number of demonstration charts in this paper, available economical data was chosen as a demonstration data set. The data is taken, as an example, from the site http://www.indexmundi.com/commodities/ where the random distributions of monthly prices on different commodities are presented. The variations of prices on gold (Au), playing a role of important strategic material, is used as 
an example. Analysis of this data is described in detail in Section 3.

In practice, for each given sequence the compression of data containing large number of points to a set of data containing less number of points needs a special procedure but in general one can recommend the following requirements:

R1. Any external factor (time, frequency, coordinate, or other) measured in general in number of the measured points should be reduced to a minimal number of 100-150 points in order to provide the value of the relative error of less than $10 \%$ (see expression $(20))$.

R2. For economical and other data associated with human activity it is natural to choose the corventional intervals as years, half-years, months, weeks, days, etc.

R3. The scaling factor, $\xi$, should lie in acceptable limits in order to keep the calculated value of the power-law parameter $v$ (associated with fractal dimensions) within the interval $0<v<3(4)$.

It is noted that these general criteria are approximate and it is expected that more definite criteria can be formulated. In this paper for the available data analyzed, these requirements were taken into account.

\subsection{Criterion of selection of the initial hypoth- \\ esis}

Analysis of solution (4) prompts to put forward the following initial hypothesis:

$$
\begin{aligned}
H_{0}(t) & =A_{0}+A_{1} t^{a_{0}}+A c_{1} y c_{1}(t)+A s_{1} y s_{1}(t), \\
y c_{1}(t) & =t^{a_{1}} \cos \left(\omega_{1} \ln t\right), y s_{1}(t)=t^{a_{1}} \sin \left(\omega_{1} \ln t\right)
\end{aligned}
$$

Here and below the same notation is kept for 4 linear unknown amplitudes; $A_{0}, A_{1}, A c_{1}$, and $A s_{1}$. The three unknown nonlinear parameters, $\alpha_{1}, \alpha_{2}$, and $\omega_{1}$, in (6) can be found by the eigencoordinates (ECs) method [11, 12] The ECs method helps to transform the curve (6) initially containing nonlinear fitting parameters into a set of straight lines, and to reduce the problem of calculation of the desired fitting parameters in the frame of the linear least square method (LLSM). The basic linear relationship (BLR) (associated with the first row of expression (9) below) obtained after three-fold integration of the differential equation (7) can be written as:

$$
D^{3} H_{0}(t)+b_{1} D^{2} H_{0}(t)+b_{2} D H_{0}(t)+b_{3} H_{0}(t)=K, D \equiv t \frac{d}{d t} .
$$

Where the parameters $\left(b_{p}, p=1,2,3\right)$ are closely associated with the roots of the cubic equation:

$$
\begin{aligned}
& r^{3}+b_{1} r^{2}+b_{2} r+b_{3}= \\
& \left(r-\alpha_{1}\right) \cdot\left(r-\alpha_{1}-i \omega_{1}\right) \cdot\left(r-\alpha_{1}+i \omega_{1}\right)=0 .
\end{aligned}
$$

Performing a triple integration of equation (7), which helps to keep the initial error in the same limit as in the function $H_{0}(t)$ ), allows finding the constants, $b_{1,2,3}$, of the cubic equation (8) by a linear procedure using, for this purpose, a stable procedure as the LLSM. The basic linear relationship (BLR) obtained after three-fold integration of the differential equation (7) can be written as:

$$
Y(t)=\sum_{s=1}^{6} C_{s} X_{s}(t) .
$$

Here the functions forming the desired BLR are determined by the following relationships

$$
\begin{aligned}
& Y(t)= H_{0}(t)-\langle\ldots\rangle, \\
& X_{1}(t)= \int_{t_{0}}^{t} H_{0}(u) \frac{d u}{u}-\langle\ldots\rangle, C_{1}=-b_{1}, \\
& X_{2}(t)= \int_{t_{0}}^{t}(\ln t-\ln u) H_{0}(u) \frac{d u}{u}-\langle\ldots\rangle, C_{2}=-b_{2}, \\
& X_{3}(t)=\int_{r_{0}}^{t}(\ln t-\ln u)^{2} H_{0}(u) \frac{d u}{u}-\langle\ldots\rangle, C_{3}=-b_{3}, \\
& \quad s=4,5,6 \\
& X_{s}(t)=\ln ^{7-5}(t)-\langle\ldots\rangle, C_{s}= \\
& C_{s}\left(K, D^{3} H_{0}\left(t_{0}\right), D^{2} H_{0}\left(t_{0}\right), D H_{0}\left(t_{0}\right)\right) .
\end{aligned}
$$

The pair of brackets in the last expressions $(10),\langle\cdots\rangle=$ $N^{-1} \sum_{j-1}^{N}(\cdots)$, defines the arithmetic mean of the neighbouring expression located on the left which should be subtracted from it. A definitive form for the constants $C_{s}=C_{s}\left[K, D^{3} H_{0}\left(t_{0}\right), D^{2} H_{0}\left(t_{0}\right), D H_{0}\left(t_{0}\right)\right]$, depending on the initial values of the derivatives in the initial point $t_{0}$, is not essential for the calculation of the desired $C_{l}$ $(i=1,2,3)$ and can be omitted. If the initial hypothesis (6) is supposed to be correct, then it can be expected that the exponents $\alpha_{0}$ and $\alpha_{1}$ should have at least the same sign and cannot be strongly deviated from each other. In the opposite case it is necessary to consider another hypothesis. The test calculations realized on model data show that the hypothesis in (6) does not provide the desired accuracy with respect to the value of the relative error (see 
expression (23) below). This means that in cases when the value of the relative error remains large, the initial hypothesis should be replaced by an alternative hypothesis which has an increased level of complexity when compared with the initial expression (6). In order to have the justified criterion that shows the conditions of replacement of (6) by another hypothesis it is necessary to consider a more complicated initial expression:

$$
\begin{aligned}
\tilde{H}_{0}(t) & =A_{0}+A_{1} t^{a_{0}}+B_{1} t^{\beta_{0}}+\sum_{p=1}^{2}\left[A c_{p} y c_{p}(t)+A s_{p} y s_{p}(t)\right] \\
y c_{p}(t) & =\exp \left(\alpha_{p} \ln (t)\right) \cos \left(\omega_{p} \ln t\right), y s_{p}(t) \\
& =\exp \left(\alpha_{p} \ln (t)\right) \sin \left(\omega_{p} \ln t\right), p=1,2 .
\end{aligned}
$$

This hypothesis contains 6 nonlinear parameters $\left(\alpha_{0}, \beta_{0}, \alpha_{1,2}, \omega_{1,2}\right)$. At first sight one has the impression that the linear procedure applied in the form of the LLSM to $H_{0}(t)$ is not applicable for this complex case. However, this idea is not true and rewriting expression (11) in the form:

$$
\begin{aligned}
\tilde{H}_{0}(t) & =h_{0}(t)+y 2(t), h_{0}(t) \\
& =A_{1} t^{a_{0}}+A c_{1} y c_{1}(t)+A s_{1} y s_{1}(t), \\
y s_{2}(t) & =A_{0}+B_{1} t^{\beta_{0}}+A c_{2} y c_{2}(t)+A s_{2} y s_{2}(t), \\
y c_{p}(t) & =\exp \left(\alpha_{p} \ln (t)\right) \cos \left(\omega_{p} \ln t\right), y s_{p}(t) \\
& =\exp \left(\alpha_{p} \ln (t)\right) \sin \left(\omega_{p} \ln t\right), p=1,2 .
\end{aligned}
$$

one can apply the ECs method for the function $y_{2}(t)$ (having the same structure as the previous function $\left.H_{0}(t)\right)$ and consider the nonlinear parameters $\left(\alpha_{0}, \alpha_{1}, \omega_{1}\right)$ as known. The initial (inoculating) values are obtained from expressions in (10). Taking into account the irvariance of the expressions relatively procedure of the $n$-fold integration $J_{n}$ :

$$
\begin{aligned}
& J_{n}(\exp (a x) \cos (b x))= \\
& K_{1, n}(a, b) \exp (a x) \cos (b x)+K_{2, n}(a, b) \exp (a x) \sin (b x), \\
& J_{n}(\exp (a x) \sin (b x))= \\
& Q_{1, n}(a, b) \exp (a x) \cos (b x)+Q_{2, n}(a, b) \exp (a x) \sin (b x),
\end{aligned}
$$

leads again to the BLR which is similar to the expression (9), but with six functions $X_{s}(t)(s=1,2, \ldots, 6)$ initially figuring in the BLR in (9), it is necessary to add three additional functions:

$$
\begin{aligned}
& X_{7}(t)=t^{a_{1}}-\langle\ldots\rangle, C_{7}, \\
& X_{8}(t)=t^{a_{1}} \cos \left(\omega_{1} \ln (t)\right)-\langle\ldots\rangle, C_{8}, \\
& X_{9}(t)=t^{a_{1}} \sin \left(\omega_{1} \ln (t)\right)-\langle\ldots\rangle, C_{9} .
\end{aligned}
$$

The modification of the BLR in (9) along with the additional expressions in (14) helps to find the desired values of three important constants, $C_{i}(i=1,2,3)$ (the values of other constants; $C_{4}, C_{5}, \ldots, C_{8}, C_{9}$; are not essential and can be omitted), and thereby it is possible to calculate the necessary nonlinear parameters; $\beta_{0}, \alpha_{2}$, $w_{2}$; in the frame of the LLSM. As it has been mentioned above in expressions (6). (11) and (12) the same notations for the linear fitting parameters are used, but in fact they are different. These linear parameters are not essential for formulating the desired criterion and therefore the usage of the same notation seems natural. Now we formulate the criterion for the applicability of the initial hypothesis (11). Let the following three ratios be defined:

$r_{p 1}=\frac{\max \left(\alpha_{0}, \alpha_{1}\right)}{\min \left(\alpha_{0}, \alpha_{1}\right)}, \quad r_{p 2}=\frac{\max \left(\beta_{0}, \alpha_{2}\right)}{\min \left(\beta_{0}, \alpha_{2}\right)}, r_{w}=\frac{\max \left(\omega_{1}, \omega_{2}\right)}{\min \left(\omega_{1}, \omega_{2}\right)}$.

The initial hypothesis (11) is applicable if the following conditions are satisfied:

$$
1<r_{p 1}, r_{p 2}<1.45,1<r_{w}<1.45 .
$$

The upper limit (1.45) of this inequality is chosen from the following condition. Being rounded off the integer value it should give again the unit value. If condition (16) is violated, then it is necessary to substitute (11) and to consider alternative hypotheses. Alternative hypotheses are considered in the Mathematical Appendix 5.

\subsection{The optimization procedure}

The third step is related to optimization of the power-law exponent, $\alpha$, and inoculating frequency that are located in the intervals:

$$
\begin{aligned}
& \min \left(\alpha_{0}, \beta_{0}, \alpha_{1}, \alpha_{2}\right)=\alpha_{\min }<\alpha<\max \left(\alpha_{0}, \beta_{0}, \alpha_{1}, \alpha_{2}\right)=\alpha_{\max } . \\
& \min \left(\omega_{1}, \omega_{2}\right)=\omega_{\min }<\omega<\max \left(\omega_{1}, \omega_{2}\right)=\omega_{\max } .
\end{aligned}
$$

If some nonlinear fitting parameter is located in the given limits $\left[p_{\min }, p_{\max }\right]$ then one can introduce the function:

$$
\begin{aligned}
& p(v)=t v+e, \\
& t=\frac{P_{\operatorname{ma}}-P_{\min }}{V_{\max }-V_{\min }}, e=\frac{P_{\min } V_{\operatorname{ma}}-P_{\max } V_{\min }}{V_{\max }-V_{\min }} .
\end{aligned}
$$

One can find the optimal value, $v_{\text {opt }}$, by minimizing the fitting function:

$$
\begin{gathered}
\begin{aligned}
& H(t, v)=A_{0}+A_{1} \exp (v(v) \ln t)+A c \cdot y c(t, v)+ \\
&+A s \cdot y s(t, v) \\
& y c(t, v)=\exp (v(v) \ln t) \cdot \cos (\omega(v) \ln t), \\
& y s(t, v)= \exp (v(v) \ln t) \cdot \sin (\omega(v) \ln t), \\
& v(v)=t_{0} v+e_{0}, \omega(v)=t_{1} v+e_{1}
\end{aligned}
\end{gathered}
$$

with respect to the value of the minimal relative error:

$$
\min (\operatorname{Re} l E r r)_{v \rightarrow v_{a p t}}=\left(\frac{S t d e v(y(t)-H(t, v))}{m e a n|y(t)|}\right)-100 \%
$$


Where $y(t)$ defines the initial signal. After realization of this optimization procedure one can obtain the optimal values of the nonlinear fitting parameters $\langle v\rangle=t_{0} v_{\text {opt }}+$ $e_{0},\langle\omega\rangle=t_{1} v_{\text {opt }}+e_{1}$.

\subsection{The final fit of the initial function}

The final hypothesis that satisfies the functional equation (3) should be presented in the form

$$
\begin{aligned}
& H_{f}(t)=A_{0}+A_{1} t^{(v)}+\sum_{k=1}^{K}\left(A c_{k} y c_{k}(t)+A s_{k} y s_{k}(t)\right) . \\
& y c_{k}(t)=\exp (\langle v\rangle \ln t)-\cos \left(2 \pi k \frac{\ln r}{\ln r}\right) . \\
& y s_{k}(t)=\exp (\langle v\rangle \ln t)-\sin \left(2 \pi k \frac{\ln t}{\ln \zeta}\right) .
\end{aligned}
$$

Here the parameters; $A_{0}, A_{1}$ and the set $A c_{k}, A s_{k}$ ( $k=$ $1,2, \ldots, K)$ denote the final set of the fitting parameters and are calculated in the frame of the LLSM. Other parameters that enter into expression (21) and the scaling equation (3) are determined as:

$$
\begin{aligned}
& \ln \xi=\frac{2 \pi}{\langle\omega\rangle}, \quad \lambda_{1}=\exp (\langle v\rangle \cdot \ln \xi),\left(\lambda_{1}>0\right), \\
& s_{0}=A_{0}-\left(1-\lambda_{1}\right), \\
& \ln \xi=\frac{\pi}{\langle\omega\rangle}, \quad \lambda_{1}=-\exp ((v\rangle \cdot \ln \xi),\left(\lambda_{1}<0\right), \\
& 2 \pi k \rightarrow \pi(2 k-1) .
\end{aligned}
$$

The second row of this expression is written for the case when the single root, $\lambda_{1}$, is negative. For this case the log-periodic function in (21) becomes anti-periodic and it is necessary to make a replacement in (21) in accordance with requirement given by the third row in (22).

It should be stressed here that the final criterion after the usage of initial criterion (16) is related to the quality of the final fitting with respect to the number of modes $K$ used for this purpose.

$$
\min (\operatorname{RelErr})_{K}=\left(\frac{S t d e v\left(y(t)-H_{f}(t, K)\right)}{\operatorname{mean}|y(t)|}\right) \cdot 100 \%
$$

This parameter should satisfy to the following requirement, $2 K+4<N$, where $N$ denotes the total number of the measured points figuring in the initial function $y\left(t_{j}\right)$ $(j=1,2, \ldots, N)$. In the opposite case where the number of unknown modes, $K$, exceeds the number of data points $N(2 K+4>N)$, the fitting procedure becomes useless. Before starting to analyse real data it is necessary to point out that in order to make the temporal variable dimensionless it is natural to normalize it to the value of the initial interval. In this case this value will coincide with the unit.

\section{Economical data (description of the distribution of prices on gold)}

In order to demonstrate the effectiveness of the fitting functions found for four cases (see Mathematical Appendix 5) describing the properties of self-similar curves, real data available on the internet was chosen. This data is freely available for the reader to repeat the calculations described in this paper. Here it is necessary to stress the following point: Example data was chosen for a given period of time and the extension of other intervals was not considered. As it follows from the method suggested; the intervals do not influence on the limits of the temporal interval (covering global draw-ups and draw-downs). It works properly and can be applicable for a wide class of time series. The content of this paper does not allow consideration of other examples and an analysis on the limits of this algorithm merits further research. The monthly distribution of prices on gold (Au), as important strategic material, is presented as the first example. The data is taken from the site http://www.indexmundi.com/commodities/, where the random behaviour of monthly prices on different commodities is presented. The given data contains 360 measured points (starting from 09/1983 and finishing on 09/2012) and covers a thirty years period. The plots presented by the Figures 1-4 demonstrate the variation of the price (that is given in kilo-US dollars) of gold for one Troy Ounce. As it follows from analysis of these figures the realization of the procedure described in Subsection 2.1 leaves these curves similar to each other. One can notice also that the compression of the initial interval in 12 times (see Figures 1 and 4 for comparison increases the corridor of uncertainty between maximal (prices up) and minimal (prices down) prices. After verification of the SS principle it becomes possible to realize the initial fit.

The fitting of the hypothesis $H_{0}(t)$ from (6) is realized with the help of the ECs method (expressions (9) and (10) in this case) which helps to apply the LLSM for the calculation of unknown non-linear parameters. This fit is shown in Figure 5 by a solid green line. The verification of criterion (15) and realization of the fit with more complicated hypothesis, $\hat{H}_{0}(t)$, from expression (11) (it is shown on Figure 5 by solid red line) shows that the influence of the corrections figuring in (11) are negligible and the behaviour of the fitting curve is determined presumably by the hypothesis, $H_{0}(t)$, from (6). This shows that, for the final fitting, one can use the simplest case 1 and the optimization and final hypotheses are determined by expressions (19) and (21). Using the optimal values of the power-law exponent $\langle v\rangle=3.07938$ and frequency $\langle\omega\rangle=1.89072$ it becomes possible to obtain the final fit 


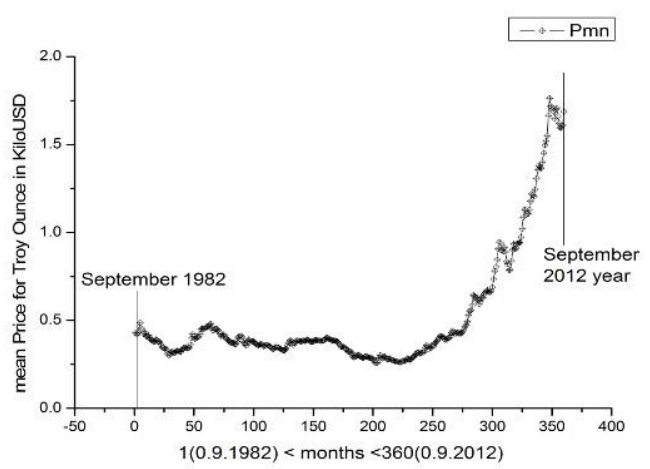

Figure 1. Initial data that show the distribution of prices of gold (for one Troy Ounce in kiloUSD). These data are taken from the site http://www.indexmundi.com/commodities/.

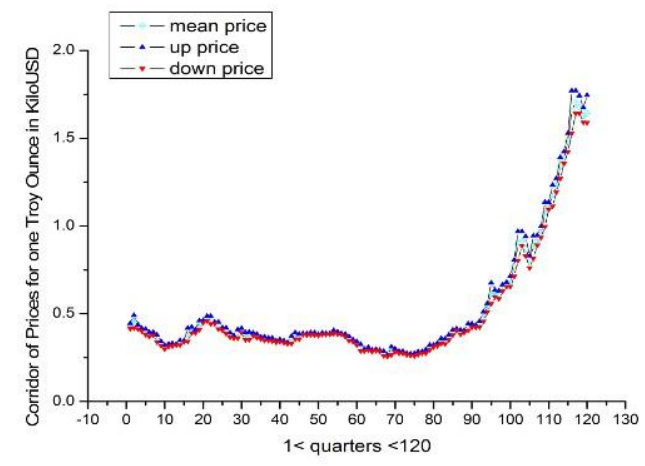

Figure 2. In the result of application of procedure described in the Subsection 2.1 (reduction to three incident points) one can take the self-similar curve showing the quarter distribution of prices. The degree of compression is equalled 3

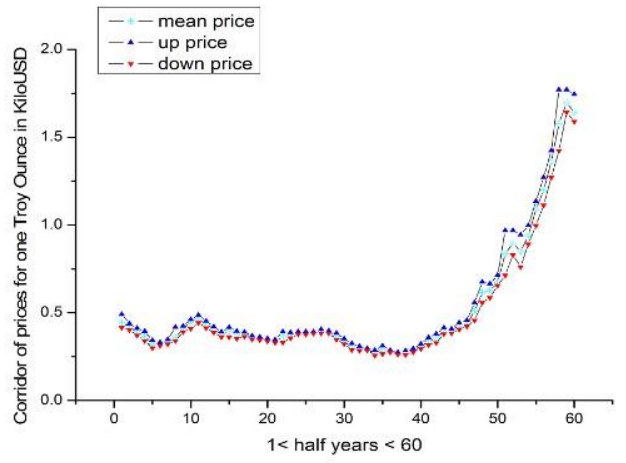

Figure 3. The property of self-similarity of the initial curve is conserved if one takes the half-year period intervals. The degree of compression is 6 (in comparison with the Figure 1).

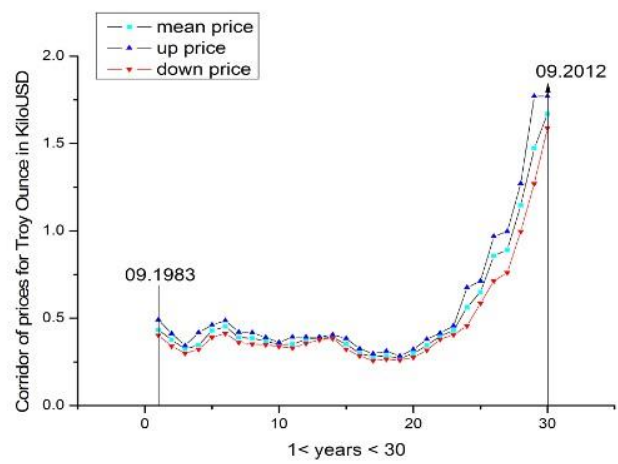

Figure 4. The self-similarity is still conserved if we realize the re- duction of twelve months points to three points character- izing a year. We note that the interval of deviation of prices from its mean value (a specific measure of uncertainty) is increased with narrowing of the initial interval. We com- pressed 12 months to one year (i.e., the degree of com- pression is 12).

with the help of the LLSM, this is shown in Figure 6 by a solid green line. During the calculation of the final fit the natural requirement that the maximal number of unknown parameters is taken into account, including the number of unknown amplitudes denoting the behaviour of the logperiodic function in (21), which cannot exceed the given number of points $N$. The maximal number of the fitting parameters for this case is determined by the condition, $(2 K+4)$. The value of $K=50$ was chosen in order to satisfy the inequality $104<N=360$. For the realization of the fit of the compressed curves (corresponding to a quarter year, half year, and one year) we take into account the following observation. The fitting curves from expressions (11) and (21) are irvariant relative to the scaling transformation, $t \rightarrow u t$. At this transformation the corresponding curves remain irvariant and only the linear parameters, $A_{0}$, $A_{1}$, together with the amplitudes of log-periodic functions are changed. In particular the amplitudes are transformed as:

$$
\begin{aligned}
& H_{f}(t u)= \\
& A_{0}+A_{1} u^{\langle v\rangle} t^{\langle v\rangle}+\sum_{k=1}^{K}\left[A \tilde{c}_{k}(u) y c_{k}(t)+A \tilde{s}_{k}(u) y s_{k}(t)\right\} \\
& A \tilde{c}_{k}(u)=A_{1} u^{\langle v\rangle}\left[\sin \left(\theta_{k}(u)\right) A s_{k}+\cos \left(\theta_{k}(u)\right) A c_{k}\right], \\
& A \bar{s}_{k}(u)=A_{1} u^{\langle(v)}\left[\cos \left(\theta_{k}(u)\right) A s_{k}-\sin \left(\theta_{k}(u)\right) A c_{k}\right], \\
& \sin \left(\theta_{k}(u)\right)=\sin \left[(2 \pi k+(a-2) \pi) \frac{\ln u}{\ln \xi}\right] \\
& \cos \left(\theta_{k}(u)\right)=\cos \left[(2 \pi k+(a-2) \pi) \frac{\ln u}{\ln \xi}\right] \\
& a=2, \quad \lambda_{1}>0, \quad a=1, \quad \lambda_{1}<0 .
\end{aligned}
$$




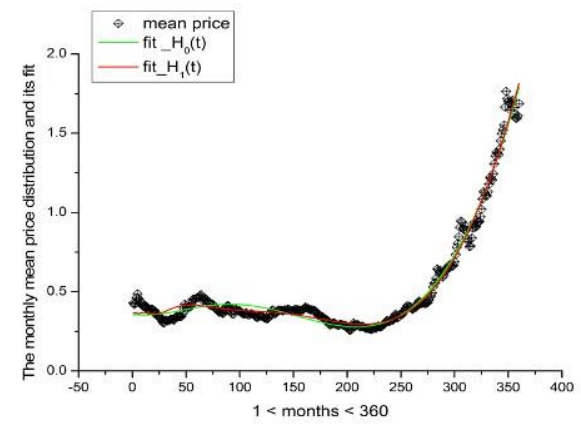

Figure 5. Here we show the realization of the stage 2 described in Subsection 2.2. The initial fit was realized with the help of the ECs method and initial hypothesis (ffrom (6) and hypothesis $H_{1}$ from expression (11) are shown by green and red solid lines, respectively. As one can notice from this plot the influence of more complicated hypothesis is not essential and so it is sufficient to use the initial hypothesis $H_{0}(t)$ corresponding to the case 1 .

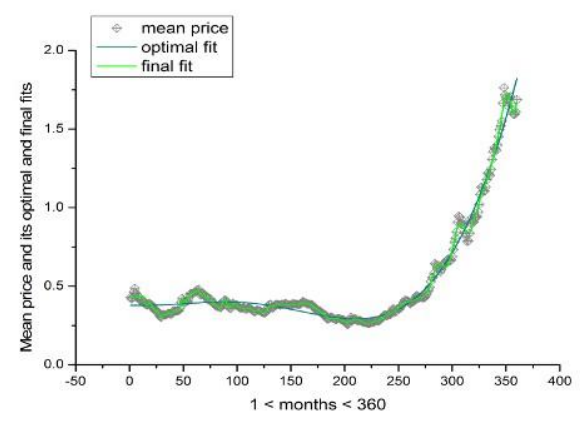

Figure 6. Here we show the optimal fit (the solid blue line) that is realized with the help of the function (18). It minimizes the value of the relative error $(9.291 \%)$ and helps to obtain the optimal values of the power-law exponent $h v i=3: 07938$ and $h \omega i=1: 89072$. The final fit (solid green line) is realized with the help of the function (20). All fitting parameters are collected in Table 1.

This observation facilitates the calculation of the unknown scaling parameter, $\xi$, figuring in the functional equation (3). For the restoration of its actual value it is supposed that it is restored from the data having maximal values of the data points (in our case from the initial data having 360 points). Owing to this irvariance, one can reduce the uncertainty in the calculation of $\ln (\bar{\xi})$ and carry-over a possible error in calculation of $\xi$ to the unknown values of amplitudes. One can realize the fitting procedure described in Section 2 and fit the rest curves describing the distribution of prices for the quarter, half-year, and one year periods. They are shown in Table 1, accordingly. For all calculated amplitudes the natural requirement, $2 K+4<N$, is taken into account. Starting from number of modes with $K=50(N=360)$, with a limited value up to $K=5(N=60)$ for the final price distribution. From this analysis one can make an important conclusion that the detailed description of a random self-similar curve (in the limits of high accuracy: (3-5\%) of the relative error) requires proper specification of the log-periodic function figuring in expression (21). From a general point of view any reliable fitting of a random curve with high accuracy (especially economic data) has a temptation to continue these data to the nearest future for its possible prediction. Predictions could be made by using the found optimal fit curve to the known data and to extend the range of its outputs beyond the measurements. This fitting function is "recognized" for the segment of a temporal interval that is supposed to be known. This type of prediction is possible if we simply continue tendencies collected in the past for the future temporal interval. Naturally, the boundaries of the sequential future interval are limited by future events that can change the tendencies "stretched out" from the known past to the future. The self-similarity principle based on the "recognized" fitting function (21) helps to continue this function into the nearest future. The results of continuation on five future points for initial, half-year and year distribution prices are presented in Figures 13 and 14. Definitely, this type of prediction can be defined as "technical forecast", nevertheless, the usage of this possibility that is given by curve (21), as an additional source of information, is important from our point of view. Analyzing the red curves depicted on Figure 13 one can notice a specific bend located on the right-hand side of these fitting curves. This bend can explain the sharp dip of prices on gold in the near future. In Figure 14 this bend is $a b$ sent but the tendency to have a sharp decreasing of prices on gold in the nearest 5 years is conserved.

\section{The mean temperature data}

Another interesting set of data tightly associated with the self-similarity principle is related with increasing of the mean temperature measured for both hemispheres of the Earth (the so-called global warming phenomenon). This data was taken from the site http://data.giss.nasa.gov/gistemp/. The curves depicted in Figures 15 and 16 include 130 years of mean temperature registrations covering the period between 1881-2011. Being compressed three times, it can be seen that these curves are self-similar. Notice Figures 16 and 17 where this compression (each three years are averaged up to one point) is shown. In comparison with the first example we do not want to compress them further because the number of data points (130) in comparison with the first case (360) 
Table 1. The table of additlonal and scaling parameters that descrbe the economical data.

\begin{tabular}{|c|c|c|c|c|c|c|c|}
\hline Number of flle & $\langle v\rangle$ & $\ln (\xi)$ & $\lambda_{1}$ & $A_{1}$ & $s_{0}$ & RelErr(\%) & $K / 2$ \\
\hline Price (1 month, 360) & 3.07938 & 3.32317 & 27814.4 & $-1.78581 E-6$ & -11985.5 & 3.15845 & 50 \\
\hline Price Mn ( $\frac{1}{4}$ year, 120$)$ & 3.10159 & 3.31473 & 29170.7 & $7.08994 \mathrm{E}-4$ & -12564.1 & 3.68348 & 25 \\
\hline Price Up ( $\frac{1}{4}$ year, 120 ) & 3.10159 & 3.31473 & 29170.7 & 0.00101 & -13025.6 & 4.65095 & 25 \\
\hline Price Down ( $\frac{1}{4}$ year, 120$)$ & 3.16822 & 3.28965 & 33601 & $1.27295 E-4$ & -13738.7 & 3.40283 & 25 \\
\hline Price $M n\left(\frac{1}{2}\right.$ year, 60$)$ & 3.14601 & 3.29796 & 32061.8 & $-3.5195 E-4$ & -13845.8 & 3.11844 & 15 \\
\hline Price Up ( $\frac{1}{2}$ year, 60$)$ & 3.16822 & 3.28965 & 33601 & $-6.87652 \mathrm{E}-$ & -15595.1 & 4.63207 & 15 \\
\hline Price Dn ( $\frac{1}{2}$ year, 60$)$ & 3.39031 & 3.20872 & 53026.3 & -289714 & -21883.4 & 2.92961 & 15 \\
\hline Price Mn (1 year, 30) & 3.21263 & 3.27314 & 36878.7 & $-9.89242 E-4$ & -17427.7 & 4.87768 & 5 \\
\hline Price Up (1 year, 30) & 3.52356 & 3.16205 & 68986.5 & $-1.91479 E-4$ & -25360.2 & 4.16153 & 5 \\
\hline Price Dn (1 year, 30) & 3.52356 & 3.16205 & 68986.5 & $1.91479 E-4$ & -25360.3 & 4.16153 & 5 \\
\hline
\end{tabular}

Comments to Table 1. The fitting parameters collected in Table 1 reflect the basic parameters of the ftnal fttting function (20) and the parameters that flgure in the scaling equation (2), along with its solution (3). Strictly speaking it is necessary to take the mean values of the columns $2,3,4$, and 6 , but they were kept as they are in order to see the varlatlons of these values with respect to the compression procedure. The last column shows the number of modes that can restore the unknown log-perlodic function with accuracy given in column 7 .

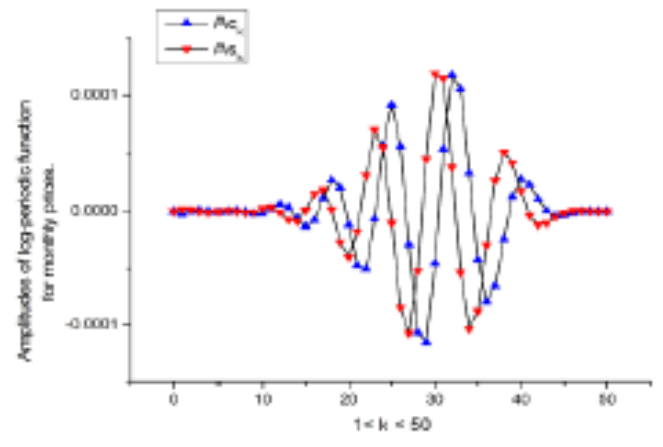

Figure 7. Besides the parameters that restore the structure of the scaling equatlon (2) we show here the distribution of the amplitudes $A c_{k}$, Ask that define the unknown log-perlodic functlon in expression (20). In order to decrease the number of the fitting parameters and in the same time to keep the acceptable accuracy for the final ftting function in the Interval (3-5\%) we choose the value of the final mode $(K)$ from the condition $(2 K+4-104<N-360)$.

is much smaller. Based on this data one can formulate another problem: to recognize the adequate hypothesis and then to fit it to the initial curve with high accuracy. In comparison with the first case the temperature fluctuations are rather high and so a possible forecast will be realized with the help of the fitting curve obtained after the optimization procedure (Subsection 2.3). The initial fit realized with hypothesis (6) and (11) leads to the following ratios: $r_{p 1}=4.54513, r_{p 2}=1.53165, r_{\mathrm{w}}=2.22379$. Figure 18 demonstrates that the influence of the second iteration (hypothesis $H_{1}(t)$ ) is essential for this case and

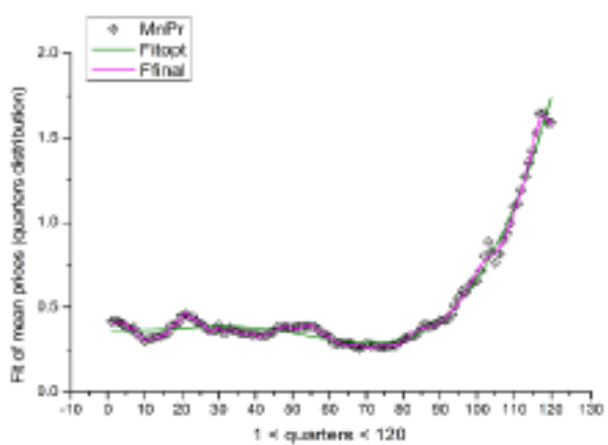

Figure 8. The final fin of the distribution of the quarter prices. The corresponding parameters are collected in Table 1. Other two curves (shown on Figure 2) are very close to each other and so their fit is not shown.

so the simple hypothesis connected with the case 1 is rejected. In accordance with definitions of these values in (15) and condition of applicability of (A11) one can conclude that the hypothesis corresponding to case 4 is preferable. The application of an alternative hypothesis corresponding to case 3 demonstrates its inconsistency and gives the high values of the fitting error at the optimal selection of the value of $K$ and so it is also rejected. Figure 19 shows the optimal fit (black solid line) realized with the help of the fitting function (A12) (where only the values of the power-law exponents are optimized) and the final fit (magenta solid line) realized with the help of the function (A13) for distribution of mean temperature measured in the northern hemisphere $(\mathrm{NH})$. The calculated 


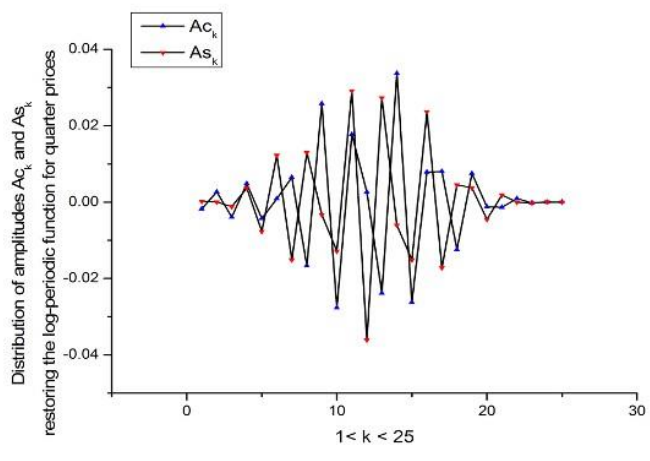

Figure 9. In comparison with Figure 7 we decrease in two times the number of corresponding amplitudes in order to keep the value of the relative error in the same limits $(3-5 \%)$ and satisfy to the requirement $(2 K+4=54<N=120)$.

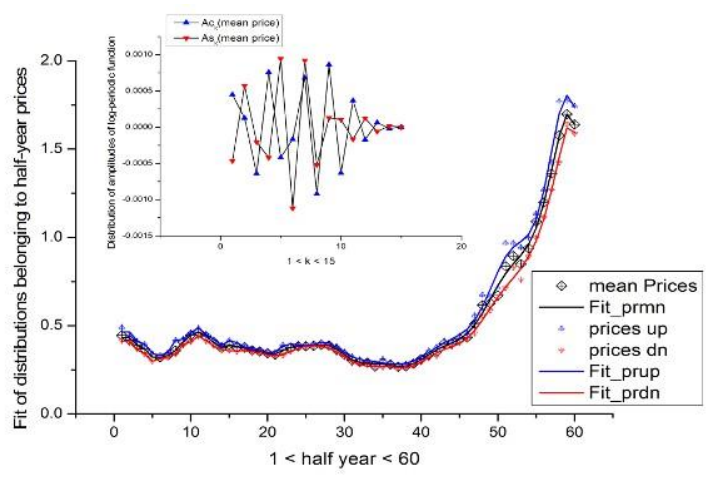

Figure 10. The final fit of the distribution of the half-year prices. The corresponding parameters are collected in Table 1. Other two curves (shown on Figure 2) (the upper and down distribution of prices) are given also. The distribution of the amplitudes for the fitting curve corresponding to mean price is given in the small frame above. Other two distributions of amplitudes for upper and down fitting curves are similar and they are notshown.

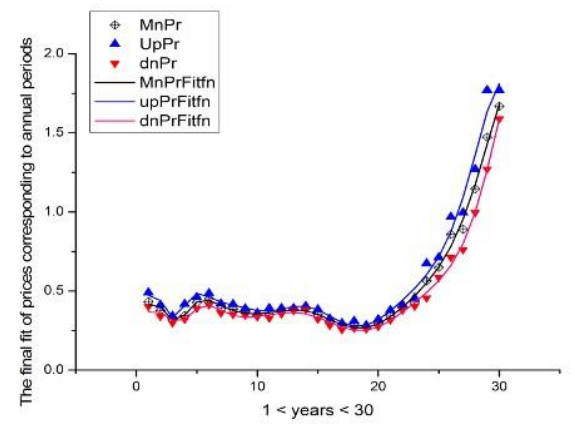

Figure 11. The final fit of the distribution of the year prices. The corresponding parameters are collected in Table 1 . The distributions of amplitudes are shown below. For this compressed curve only 5 amplitudes are necessary.

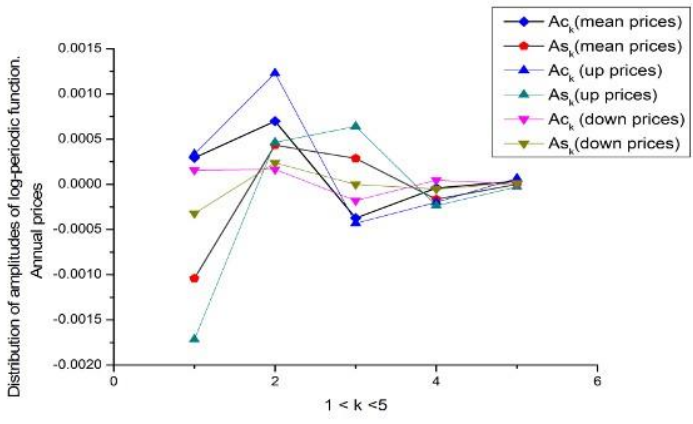

Figure 12. In comparison with Figures 7 and 9 we keep the number of modes equaled $K=5$. This number is sufficient for keeping the value of the relative error in the limits (3-5\%) and for satisfaction of the requirement $(2 K+4=14<$ $N=60)$.

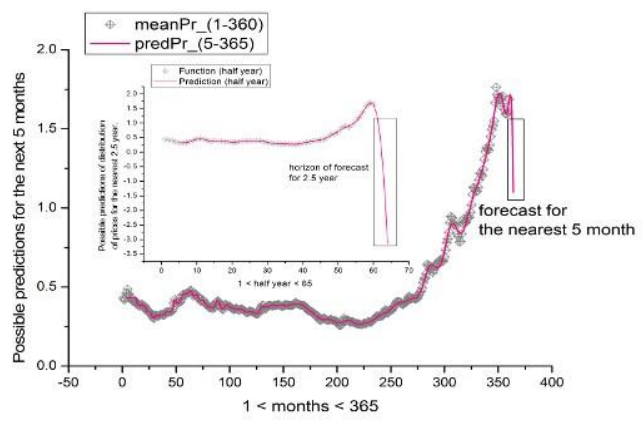

Figure 13. What is happened if we continue the fitting functions calculated for 1 month and half-year (given in the small frame) for the 5 points up? It is interesting to note that these curves demonstrate the essential decreasing of prices on gold in the nearest future.

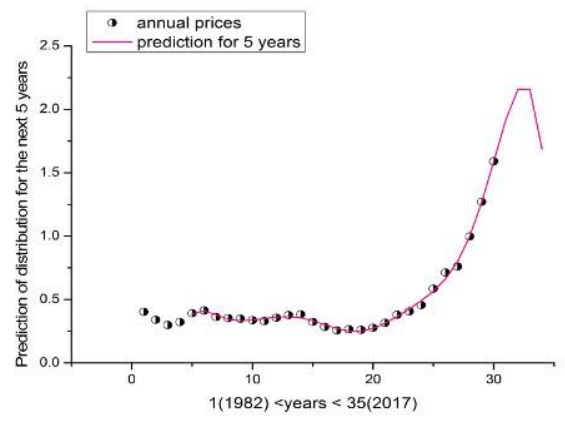

Figure 14. It is instructive to see that the distribution of the annual prices also has a tendency to decrease these prices on the nearest 5 years. For the first two curves depicted on the previous figure one can explain the "decreasing" tendency by existence of a specific bend that is clearly seen on the right-hand of these curves. But the natural bend for the curve depicted here is clearly absent but nevertheless the tendency to decreasing of the prices for the nearest 5 years is conserved. 
values of the fitting parameters are collected in Table 2. The distributions of the amplitudes that define the couple of log-periodic functions are given in Figure 20. We consider that these distributions (specifying the amplitudefrequency response (AFR/)) can serve as a key point of the whole random process studied. In the frame of the same hypothesis (case 4) one can fit the data describing the distribution of mean temperature in the southern hemisphere (SH). The final fit is shown in Figures 21 and 22, which both show strong agreement between the sample data and the fitted curve. For this, the highest value of $K$ was used from the condition, $4 K+4 \cong N=43$; all fitting parameters for these cases are collected in Table 2 . In order to save place for discussion we decided not to show the fit of the compressed functions (shown above on Figures 16 and 17). The final fit looks similar as it is given on Figures 19 and 21 realized for the initial temperature distributions. The fitting parameters associated with this procedure are kept in Table 2 also. After realization of this procedure one can say that the restored scaling equation, describing the distribution of mean temperature in both Earth's hemispheres, coincides with expression (A15). All parameters of this equation are given in Table 2 . It is natural to pose the following question: what is the basic role of this fitting function (found from the SS principle) if the concrete model is absent? We see the basic role of this fitting function (besides the restoration of the fitting parameters of the proper self-similar process) lies in its self-similar organization and in the possibility of "technical forecasting". Based on the information about the accurate behaviour of this function in the given segment of time one can try to continue this function into the period of time that can be associated with a future event. In the case of mean temperature distribution data one can notice that they are very "noisy" (the initial value of relative error achieves 45-50\%) and so for forecasting purposes one can use the optimal fitting function that accurately describes the trend of this distribution. Figures 23 and 24 demonstrate the results of this "technical forecast" for optimal trends obtained for distributions of mean temperature for $\mathrm{NH}$ and $\mathrm{SH}$, correspondingly. It is interesting to note that we observe two opposite tendencies. In the $\mathrm{NH}$ the forecast predicts a tendency to global cooling in the next 10 years, while there is a global warming trend forecasted for the $\mathrm{SH}$. These tendencies are conserved for the compressed curves also and they give the same forecast for the next 27 years. This forecast is preliminary. nevertheless, it is obtained from the accurate fitting of the curves that cover a rather long period of temperature observations. From this point of view a simple mechanical continuation of a random curve into the future should be corrected (or justified) by corrections of experts working

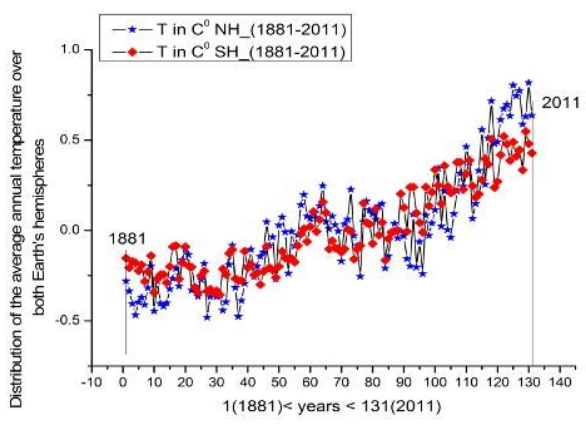

Figure 15. The distribution of mean temperature (measured in $\mathrm{C}$ ) for both Earth's Hemispheres. The data cover 130 year period of registration of mean temperature.

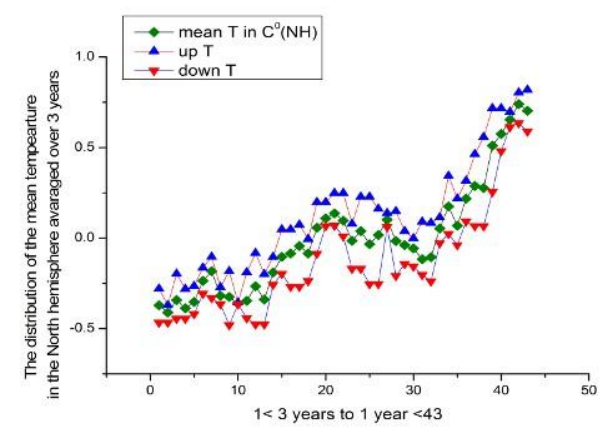

Figure 16. If one can realize the reduction to three incident points then we obtain the curve similar to the curve depicted on the previous figure. This reduction is realized for the mean temperature belonging to the North hemisphere (NH). The temperatures Tup and Tdn show the limits of deviation of the central curve from its mean position.

professionally in this field.

Comments to Tables 2 and 3: The fitting parameters collected in these Tables reflect the basic parameters of the final fitting function (A13) and the parameters figuring in the scaling equation (A15) and its solution (A14). The $8^{\text {th }}$ column in Table 2 shows the number of modes that can restore the unknown log-periodic function with accuracy given in column 7 . We should note here that increasing the number of modes up to the limiting value $4 K+4 \cong N$ sharply increases the accuracy (see column 7 in Table 2) and makes the fitting function practically exact.

\section{The basic results and discussion of further possibilities}

The two examples illustrated in this paper show that the presented method provides very good fits for datasets with 
Table 2. The table of additional and scaling parameters that describe the mean temperature data $T\left({ }^{\circ} \mathrm{C}\right)$ (the 1st part).

\begin{tabular}{lccccccc}
\hline Number of fle & $h v_{1} i$ & $\ln (\xi)$ & $A_{1}$ & $A_{0}$ &, & $\operatorname{RelErr}(\%)$ & $K / 2$ \\
\hline \hline T mn (NH) 130 & 1.76572 & 0.32278 & $4.59067 \mathrm{E}-4$ & -0.37172 & -4.34845 & 5.26015 & 31 \\
T mn (NH) (43) & 1.24181 & 0.32278 & 0.00726 & -0.39043 & -4.34845 & $1.53648 \mathrm{E}-6$ & 5 \\
T up (NH) (43) & 1.39462 & 0.32278 & 0.02812 & -0.33276 & -4.34845 & $1.82013 \mathrm{E}-6$ & 5 \\
T dn (NH) (43) & 0.7179 & 0.32278 & 0.07095 & -0.57355 & -4.34845 & $3.10656 \mathrm{E}-7$ & 5 \\
T mn (SH) 130 & 0.73562 & 0.51028 & 0.0161 & -0.48081 & -5.402 & 3.26819 & 3 \\
T mn (SH) (43) & 0.54818 & 0.51028 & 0.07595 & -0.12483 & -5.402 & $2.67959 \mathrm{E}-12$ & 5 \\
$T$ up (SH) (43) & 0.43572 & 0.51028 & 0.09562 & 0.05575 & -5.402 & $1.68926 \mathrm{E}-12$ & 5 \\
& & & & & & &
\end{tabular}

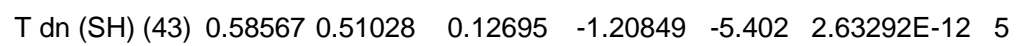

Table 3. The table of additional and scaling parameters that describe the mean temperature data $T\left({ }^{\circ} \mathrm{C}\right)$ (the 2nd part).

\begin{tabular}{ccccccc}
\hline Number of fle & $h v_{2} i$ & $A_{2}$ & $b_{2}$ & $b_{1}$ & $b_{0}$ & $s_{0}$ \\
\hline \hline T mn (NH) 130 & 7.47739 & $3.36388 \mathrm{E}-4$ & -2915.23 & -13669.9 & -125878 & -10.0722 \\
T mn (NH) (43) & 5.8845 & -0.02704 & -461.835 & -1205.18 & -6103.54 & -11.2439 \\
T up (NH) (43) & 6.34909 & -0.21924 & -62.4825 & -112.623 & -368.852 & -19.0733 \\
T dn (NH) (43) & 4.29161 & -0.02515 & -93.6704 & -144.359 & -418.556 & -16.0342 \\
T mn (SH) 130 & 0.11018 & 0.01649 & 2.04037 & -2.61815 & -7.20148 & -19.4399 \\
T mn (SH) (43) & -0.19663 & 0.00769 & 1.66488 & -1.78249 & -2.94034 & -6.04195 \\
T up (SH) (43) & -0.38072 & 0.01732 & 1.59147 & -1.6495 & -1.8612 & 2.11418
\end{tabular}

$\operatorname{Tdn}(\mathrm{SH})(43) \quad-0.13527 \quad 0.00487 \quad 1.62114-1.66886-3.25222-72.5342$

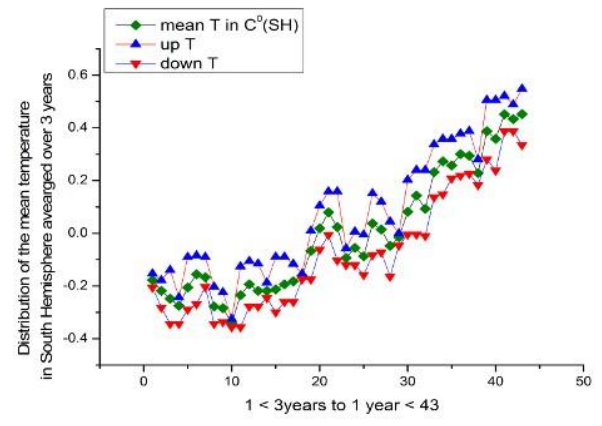

Figure 17. These plots demonstrate the reduction to three points for the mean temperature belonging to South Hemisphere (SH). As before the temperatures the curves Tup and Tdn demonstrate the limits of deviation of the central curve from its mean position.

different characteristics: $(i)$ monthly gold prices and (ii) average annual temperature on Earth surface. Both series are nonstationary and display a power-law trend while also having a random trend that changes over time. In the former, there are no substantial overall up-down monthly oscillations, which are present in the latter dataset. This second dataset is characterized by up-down annual deviations (volatility) and has visually a linear time trend

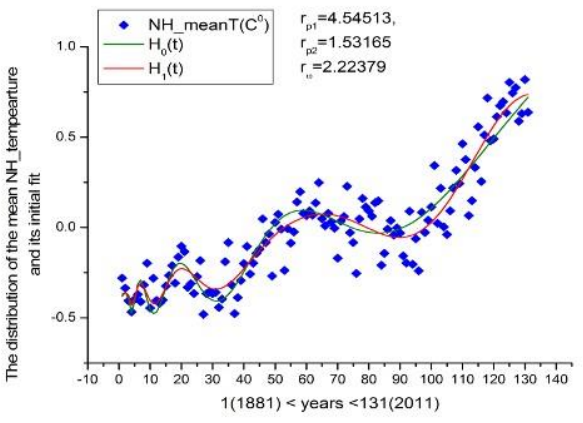

Figure 18. This plot demonstrates the selection of the proper hypothesis. The influence of the fitting function (from (11)) is essential in comparison with the function (from (6)). The values of the ratios given above on this figure and expression (A11) help to choose the proper hypothesis corresponding to case 4 .

for oscillations of the mean temperature for the $\mathrm{SH}$ (but again expressed by a posynomial function containing real and complex-conjugated power-law exponents), at least in comparison with the gold price time series. In general, time series trends reflecting the low-frequency oscillations in time can be classified as deterministic or stochastic (random), but in both cases the time series is said to be nonstationary. In the former case, stationarity can be in- 


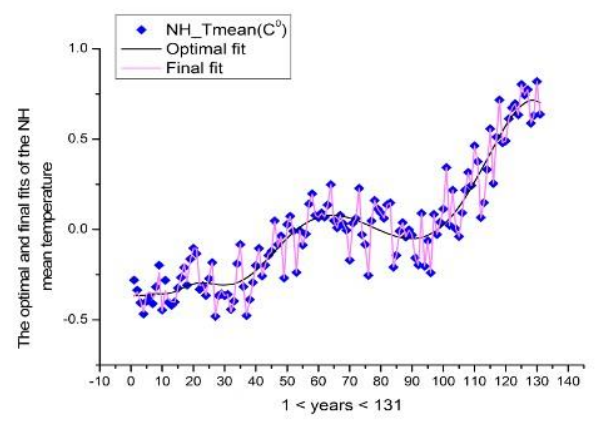

Figure 19. The optimal (solid black line) and the final fit that are obtained in the frame of hypotheses (A12) and (A13). The values of the fitting parameters are collected in Table 2 .

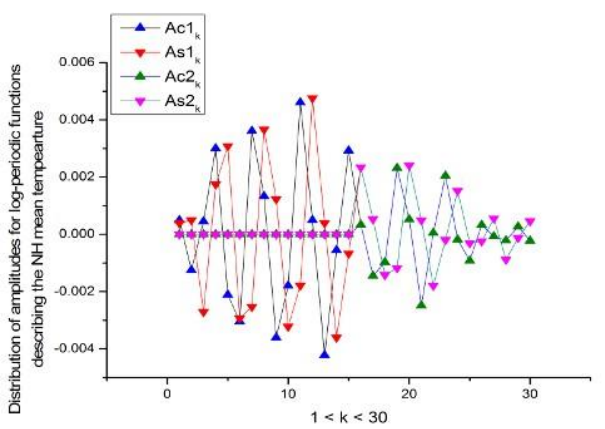

Figure 20. Distributions of amplitudes of two-log periodic functions that enter to expression (A13). They describe the distribution of $T\left({ }^{\circ} \mathrm{C}\right)$ in the $\mathrm{NH}$. Namely, these specific amplitude-frequency responses (AFRs) describing the behavior of initial random functions depicted on Figure 15 determine the ground of the whole random process studied.

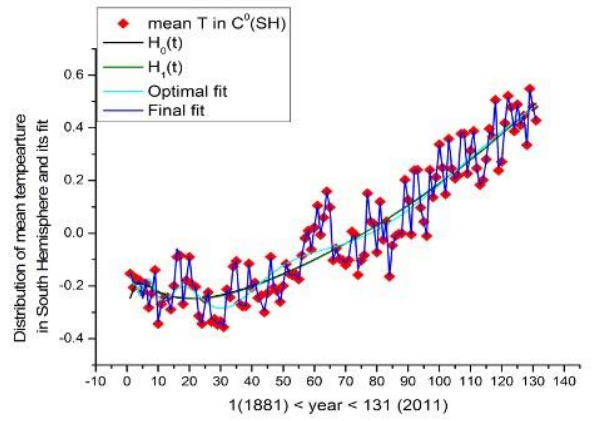

Figure 21. The optimal (solid cyan line) and the final fit (solid blue line) that describe the distribution of mean temperature in $\mathrm{SH}$. This high quality fit is realized again with the help of hypotheses (A12) and (A13). The values of the fitting parameters are collected in Table 2 .

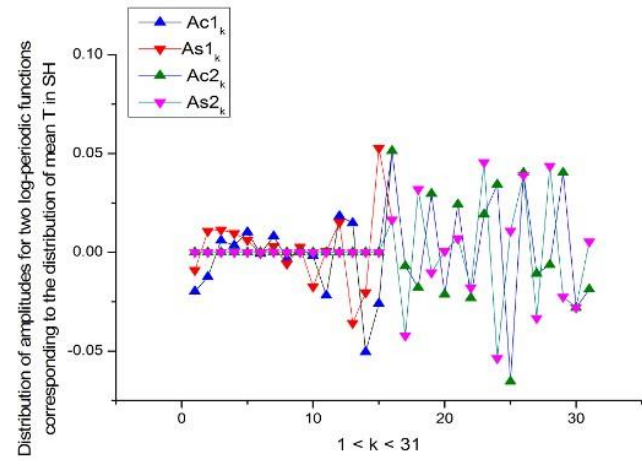

Figure 22. Distributions of amplitudes of two-log periodic functions that enter to expression (A13). These distributions (serving as the specific AFRs) describe the distribution of $T\left({ }^{\circ} \mathrm{C}\right)$ in $\mathrm{SH}$ (see previous figure).

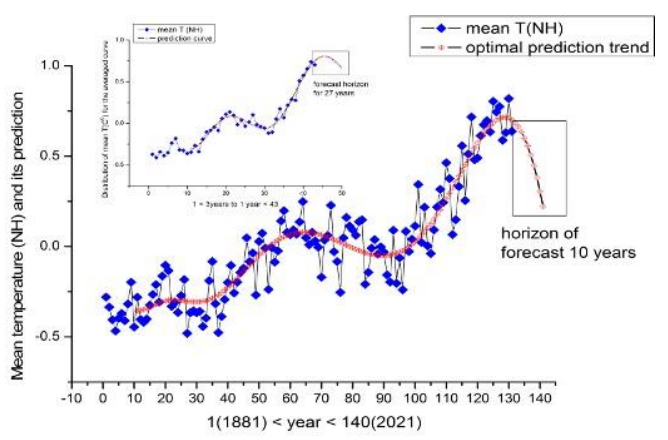

Figure 23. Distribution of mean temperature for $\mathrm{NH}$ and its forecast for the nearest 10 years. In accordance with this "prediction" we should expect the tendency to general cooling. The same tendency is observed for the compressed curve (placed in the small frame above) if we continue this curve on the nearest 27 years.

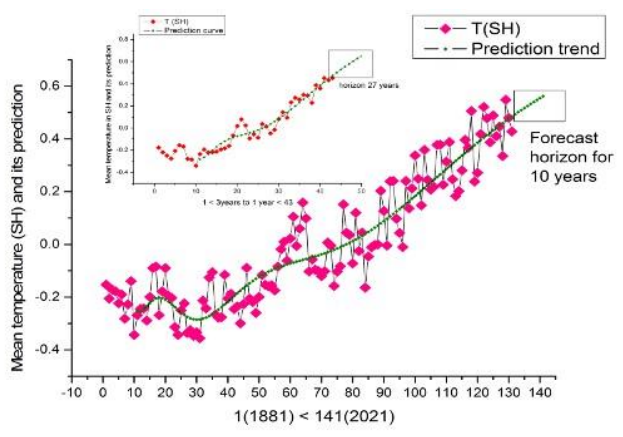

Figure 24. Distribution of mean temperature for $\mathrm{SH}$ and its forecast for the nearest 10 years. In contrast with the previous "prediction" we should expect the tendency to general warming. The same tendency is observed for the compressed curve (placed in the small frame above) if we continue this curve on the nearest 27 years. 
duced by modeling explicitly the time series as a continuous function of time (linear, quadratic, etc.). In the latter case, stationarity is only obtained by differencing the original time series. It reminds the case of the random walk where the first difference of a nonstationary time series is white noise.

In most cases, a nonstationary time series contains both deterministic and stochastic trends [13-16]. In order to determine whether stochastic trends are present, most statistical tests use a linear time trend (or, at most, a quadratic one) to capture any existing deterministic trend. The remaining is assumed to be part of the stochastic time trend (see, infer alia [17, 18]). These tests, known as unit root tests, are designed to capture only real roots. The simplicity of these tests in dealing with the deterministic trends can pose several problems because the researcher may wrongly assign to the stochastic (random) component of the time series a part that has, in reality, a deterministic behaviour. This can be easily seen from our examples. In the case of the gold price series, if a linear deterministic trend is used to test for a unit root, it seems likely that part of the "remaining" stochastic (random) trend incorporates deterministic information that we do not treat as such. The result is: wrong conclusion about the weight of the stochastic component in explaining the behavior of the variable and imprecise forecasts obtained out of this wrong model. Similarly, for the annual temperature dataset, the researcher may wrongly assign to the stochastic component the observed oscillations that can be, otherwise, captured by a properly modeled deterministic function. If this is the case, the spurious regression problem posed by [19] might be analyzed in a totally different context, since control of the stochastic component of time series would be much less problematic.

Analysis of these results based on the fitting functions found from SS principle allows us to put forward the following idea. For the fitting of different dataset having initially a random behavior we had before only one type of the fitting functions. They followed from the justified model and help to reduce $N$ initial data points to a few stationary parameters that describe the general features of the time series under analysis. In the case of success the researchers can identify these strongly-correlated relationships as laws of nature. Any other transformations realized with initial sequence (like the Fourier, numerous wavelet and other transforms) can be considered as a comvenient presentation of the initial data in order to receive an additional source of information. The SS principle gives a researcher another possibility to fit the sufficiently large initial data points. The fitting functions that follow from this principle occupy an intermediate position between the laws (that contain minimal number of the fitting parameters $P_{\min }$ ) and transformations, where the number of data points $N$ close to number of parameters that present of the initial data set in another presentation (frequency, number of moments and etc.). As it has been shown in this paper the following inequality is used:

$$
P_{m / n}<2(4) K+4(7)<N
$$

where $P_{\min }$ is the minimal number of the fitting parameters corresponding to the recognized true hypothesis (model). parameter $K$ denotes a number of log-periodic modes figuring in the recognized (identified) hypothesis. The numbers that are outside of the parenthesis correspond to the hypothesis 1 (expression (21)) and 2 (expression (A2)). while the numbers inside correspond to hypotheses 3 (A7) and 4 ((A12)). Recently this specific reduction for the case of decomposition of a random function to the Prony's spectrum was proven $[20,21]$. This proof introduced a method to differentiate an initial randomness and separate some stationary nonlinear fitting parameters together with their linear amplitudes from the rest of random parameters $(K)$ that describe the contribution of log-periodic amplitudes in expressions mentioned above ((21), (A2), (A7) and (A12)). One can conclude that the mathematical formulation of any general principle (that is considered as a source of additional information) contains some form of fitting function. These fitting functions could play their important role in analysis of different random sequences, when the strongly-correlated relationships (defined usually as natural laws) are remained unknown. For example, the linear principle of the strongly-correlated variables (LPCV) formulated in paper [22] allows to find new solutions of the Prony's problem [20, 21] and realize the decomposition (together with simultaneous reduction) of a random function having a multi-periodic structure. New laws formulated in $[20,21]$ for the strongly-correlated systems helped to realize this nontrivial decomposition.

It is in the intention for there to be a continuation and expansion on the work presented in this paper, namely in other alternative hypotheses associated with SS principle that can help to solve the problems of diagnostics, control of randomness, and fluctuation metrology, which are tightly associated with the behavior of complex systems on different levels of their organization. The recent example of consideration of the feedback control for complex systems based on the Prony's spectroscopy is considered in paper [23].

\section{Appendix A Mathematical Appendix}

In this section we describe in brief other hypotheses that can be served as alternative variants to replace the sim- 
plest, defined by (19). It should be stated that the initial hypothesis (11) for all cases considered below and the basic steps described in the second section are conserved. The optimization hypotheses and the functions used for the final fitting are changed. Only the conditions of applicability, optimization and final hypothesis are shown for each case, correspondingly.

\section{A.1. Case 2: two complex-conjugated roots}

The condition of applicability:

$$
1<r_{p 1}, r_{p 1}<1.45, r_{w}>2.45, r_{w} \neq 2 .
$$

Optimization hypothesis:

$$
\begin{aligned}
& H(t, v)=A_{0}+A_{1} t^{v[v]}+\sum_{p=1}^{2}\left[A c_{p} y c_{p}(t, v)+A s_{p} y s_{p}(t, v)\right] \\
& y c_{p}(t, v)=\exp (v(v) \cdot \ln t) \cos \left(\omega_{p} \ln t\right), \\
& y s_{p}(t, v)=\exp (v(v)-\ln t) \sin \left(\omega_{p} \ln t\right) .
\end{aligned}
$$

Final hypothesis $(2 K+2<N$, where $N$ is the number of the points in initial sequence)

$$
\begin{aligned}
& H_{f}(t)=A_{0}+A_{1} t^{(v)}+\sum_{k=1}^{K}\left[A c_{k} y c_{k}(t)+A s_{k} y s_{k}(t)\right], \\
& y c_{k}(t)=\exp (\langle v\rangle \cdot \ln t) \cos \left[(2 \pi k+\phi) \frac{\ln t}{\ln \xi}\right] \\
& y s_{k}(t)=\exp (\langle v\rangle \cdot \ln t) \sin \left[(2 \pi k+\phi) \frac{\ln t}{\ln \xi}\right] .
\end{aligned}
$$

The parameters $\ln \xi$ and $\varphi$ figuring in (A2) are found from equations:

$$
\begin{aligned}
& \omega_{1} \ln \xi=2 \pi+\phi, \quad \omega_{2} \ln \xi=4 \pi+\phi, \\
& \ln \xi=\frac{2 \pi}{\omega 2-\omega_{1}}, \quad \phi=2 \pi\left(\frac{2-r_{\alpha}}{r_{\alpha}-1}\right) .
\end{aligned}
$$

The deficient parameters of the scaling equation:

$$
\begin{aligned}
& S\left(t \xi^{2}\right)=a_{1} S(t \xi)+a_{0} S(t)+s_{0}, \\
& |\lambda|=\exp ((v\rangle \ln \xi), a_{1}=2|\lambda| \cdot \cos \phi, a_{0}=-|\lambda|^{2}, \\
& s_{0}=A_{0} \cdot\left(1-a_{1}-a_{0}\right) .
\end{aligned}
$$

\section{A.2. Case 3: two real roots}

The condition of applicability:

$$
r_{p 1}, r_{p 2}>1.45,1<r_{w}<2.45 \text {. }
$$

Optimization hypothesis:

$$
\begin{aligned}
H(t, v)= & A_{0}+A_{1} t^{v_{1}(v)}+A_{2} t^{v_{2}(v)} \\
& +\sum_{p=1}^{2}\left[A c_{p} y c_{p}(t, v)+A s_{p} y s_{p}(t, v)\right], \\
y c_{p}(t, v)= & \exp \left(v_{p}(v)-\ln t\right) \cos (\omega(v) \ln t), \\
y s_{p}(t, v)= & \exp \left(v_{p}(v)-\ln t\right) \sin (\omega(v) \ln t) .
\end{aligned}
$$

Final hypothesis $\left(\lambda_{1}, \lambda_{2}>0\right.$ and $4 K+7<N$, where $N$ is the number of the points in initial sequence)

$$
\begin{aligned}
H_{f}(t)= & A_{0}+A_{1} t^{\left\{v_{1}\right\}}+\sum_{k=1}^{K}\left[A c_{k}^{(1)} y c_{k}^{[1]}(t)+A s_{k}^{(1)} y s_{k}^{(1)}(t)\right] \\
& +A_{2} t^{\left(v_{2}\right\rangle}+\sum_{k=1}^{K}\left[A c_{k}^{(2)} y c_{k}^{(2)}(t)+A s_{k}^{[2]} y s_{k}^{(2)}(t)\right] \\
y c_{k}^{[p]}(t)= & \exp \left(\left\langle v_{p}\right\rangle-\ln t\right) \cos \left[(2 \pi k) \frac{\ln t}{\ln \xi}\right] . \\
y s_{k}^{(p)}(t)= & \exp \left(\left\langle v_{p}\right\rangle \cdot \ln t\right) \sin \left[(2 \pi k) \frac{\ln t}{\ln \xi}\right] .
\end{aligned}
$$

The parameter $\ln \xi$ figuring in $(A B)$ is found from equations:

$$
\begin{aligned}
& \ln \xi=\frac{2 \pi}{\langle\omega\rangle} . \text { If } r_{w} \cong 2 \text { then } \lambda_{1}<0, \ln \xi=\frac{\pi}{\langle\omega\rangle}, \\
& y c_{k}^{(1)}=\exp \left(\left(v_{1}\right\rangle \ln t\right) \cos \left[\pi \cdot(2 k-1) \frac{\ln t}{\ln \xi}\right] . \\
& y s_{k}^{(1)}=\exp \left(\left(v_{1}\right\rangle \ln t\right) \sin \left[\pi \cdot(2 k-1) \frac{\ln t}{\ln \xi}\right] .
\end{aligned}
$$

The deficient parameters of the scaling equation:

$$
\begin{aligned}
& S\left(t \xi^{2}\right)=a_{1} S(t \xi)+a_{0} S(t)+s_{0}, \\
& \left|\lambda_{1,2}\right|=\exp \left(\left(v_{1}, 2\right\rangle \ln \xi\right), a_{1}=\lambda_{1}+\lambda_{2}, a_{0}=-\lambda_{1} \lambda_{2}, \\
& s_{0}=A_{0} \cdot\left(1-a_{1}-a_{0}\right) .
\end{aligned}
$$

\section{A.3. Case 4: two complex-conjugated roots and one real root}

The condition of applicability:

$$
r_{p 1,} r_{p 2}>1.45, r_{w}>2.45
$$

Optimization hypothesis:

$$
\begin{aligned}
H(t, v)= & A_{0}+A_{1} t^{v_{1}(v)}+A_{2} t^{v_{2}(v)} \\
& +\sum_{p=1}^{2}\left[A c_{p} y c_{p}(t, v)+A s_{p} y s_{p}(t, v)\right], \\
y c_{p}(t, v)= & \exp \left(v_{p}(v) \cdot \ln t\right) \cos \left(\omega_{p} \ln t\right) . \\
y s_{p}(t, v)= & \exp \left(v_{p}(v) \cdot \ln t\right) \sin \left(\omega_{p} \ln t\right) .
\end{aligned}
$$


Final hypothesis $(4 K+7<N$ where $N$ is the number of the points in initial sequence)

$$
\begin{aligned}
& H_{f}(t)=A_{0}+A_{1} t^{\left[v_{1}\right\}}+\sum_{k=1}^{K}\left[A c_{k}^{(1)} y c_{k}^{(1)}(t)+A s_{k}^{(1)} y s_{k}^{(1)}(t)\right] \\
& +A_{2} f^{\left[V_{2}\right]}+\sum_{k=1}^{K}\left[A c_{k}^{(2)} y c_{k}^{(2)}(t)+A s_{k}^{(2)} y s_{k}^{(2)}(t)\right] . \\
& y c_{k}^{(1)}(t)=\exp \left(\left\langle v_{1}\right\rangle \cdot \ln t\right) \cos \left[(2 \pi k+\phi) \frac{\ln t}{\ln \xi}\right] \text {. } \\
& y s_{k}^{[1]}(t)=\exp \left(\left\langle v_{1}\right\rangle \cdot \ln t\right) \sin \left[(2 \pi k+\phi) \frac{\ln t}{\ln \tau}\right] \text {. } \\
& y c_{k}^{(2)}(t)=\exp \left(\left(v_{2}\right\rangle \cdot \ln t\right) \cos \left[\pi(2 k+(a-2)) \frac{\ln t}{\ln }\right] . \\
& y s_{k}^{[2]}(t)=\exp \left(\left(v_{2}\right\rangle \cdot \ln t\right) \sin \left[\pi(2 k+(a-2)) \frac{\ln t}{\ln l}\right] \text {. }
\end{aligned}
$$

The parameters $\ln \xi, \varphi$ and the roots are found from equations:

$$
\begin{aligned}
& \ln \xi=\frac{a_{\pi}}{w_{2}}, \phi=-\pi\left(2-\frac{a}{r_{\omega}}\right), \\
& |\lambda|=\exp \left(\ln \xi \cdot\left\langle v_{1}\right\rangle\right), \lambda_{2}=\exp \left(\ln \xi \cdot\left\langle v_{2}\right\rangle\right), \\
& \lambda_{2}=(-1)^{a}\left|\lambda_{2}\right|, \quad a=2,1 .
\end{aligned}
$$

The deficient parameters of the scaling equation:

$$
\begin{aligned}
& S\left(t \xi^{3}\right)=a_{2} S\left(t \xi^{2}\right)+a_{1} S(t \xi)+a_{0} S(t)+s_{0}, \\
& a_{2}=2|\lambda| \cdot \cos \phi+\lambda_{2}, \\
& a_{1}=-\left.2|\lambda| \lambda\left|\lambda_{2} \cos \phi-\right| \lambda\right|^{2}, \\
& a_{0}=|\lambda|^{2} \cdot \lambda_{2} \\
& s_{0}=A_{0} \cdot\left(1-a_{2}-a_{1}-a_{0}\right) .
\end{aligned}
$$

\section{References}

[1] J. Kwapien, S. Drozdz, Phys. Rep. 515, 115 (2012)

[2] A. Yasutomi, Physica D 82, 180 (1995)

[3] J. Duffy, J. Ochs, Am. Econ. Rev. 89, 847 (1999)

[4] P. Howlett, R. Clower, J. Econ. Behav. Organ. 41, 55 (2000)
[5] J. Feder, Fractals, Plenum Press (New York and London, 1988)

[6] H. Sheng, Y. Chen, T. Qui, Fractional Processes and Fractional-Order Signal Processing, Springer-Verlag (NY, Heidelberg, London, 2012)

[7] R. R. Nigmatullin, G. Smith, Physica A 320, 291 (2003)

[8] R. R. Nigmatullin, Commun. Nonlinear Sci. 15, 637 (2010)

[9] D. Sornette, Phys. Rep. 297, 239 (1998)

[10] J. Voigt, The Statistical Mechanics of the Financial Markets, 3rd edition (Springer-Verlag. BerlinHeidelberg, 2005)

[11] R. R. Nigmatullin, J. AppL. Magn. Reson. 14, 601 (1998)

[12] R. R. Nigmatullin, Physica A 285, 547 (2000)

[13] R. Menezes, N. B. Ferreira, D. A. Mendes, Nonlinear Dynam. 44, 359 (2006)

[14] J. T. Machado, G. M. Duarte, F. B. Duarte, Nonlinear Dynam. 63, 611 (2011)

[15] J. T. Machado, F. B. Duarte, G. M. Duarte, Commun. Nonlinear Sci. 16, 4610 (2011)

[16] J. T. Machado, G. M. Duarte, F. B. Duarte, Int. J. Bifurcat. Chaos 22, 1250249 (2012)

[17] D. A. Dickey, W. A. Fuller, Econometrica 49, 1057 (1981)

[18] D. Kwiatkowski, P. Phillips, P. Schmidt, Y. Shin, J. Econometrics 54, 159 (1992)

[19] C. W. J. Granger, P. Newbold, J. Econ. 2, 111 (1974)

[20] R. R. Nigmatullin, The Journal of Applied Nonlinear Dynamics 1, 173 (2012)

[21] R. R. Nigmatullin, The Journal of Applied Nonlinear Dynamics 1, 207 (2012)

[22] R. R. Nigmatullin, Phys. Wave Phenom.16, 119 (2008)

[23] R. R. Nigmatullin, W. Zhang, Commun. Nonlinear Sci. 18,547 (2013) 